\title{
Lignin-incorporated nanogel serving as an antioxidant biomaterial for wound healing
}

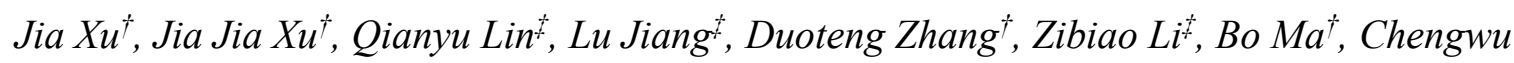

Zhang $^{\dagger}$, Lin Li*广, Dan Kai*t, Hai-Dong Yu*广s, Xian Jun Loh

${ }^{\dagger}$ Key Laboratory of Flexible Electronics (KLOFE) \& Institute of Advanced Materials (IAM), Jiangsu National Synergetic Innovation Center for Advanced Materials (SICAM), Nanjing Tech University (Nanjing Tech), 30 South Puzhu Road, Nanjing, 211816, P.R. China

Institute of Materials Research and Engineering (IMRE), A*STAR, 2 Fusionopolis Way, \#0803 Innovis, Singapore 138634

${ }^{\S}$ Frontiers Science Center for Flexible Electronics, Xi' an Institute of Flexible Electronics (IFE) and Xi'an Institute of Biomedical Materials \& Engineering, Northwestern Polytechnical University, 127 West Youyi Road, Xi'an 710072, P. R. China

*Corresponding authors. E-mail address: iamlli@njtech.edu.cn; iamhdyu@njtech.edu.cn; kaid@imre.a-star.edu.sg 

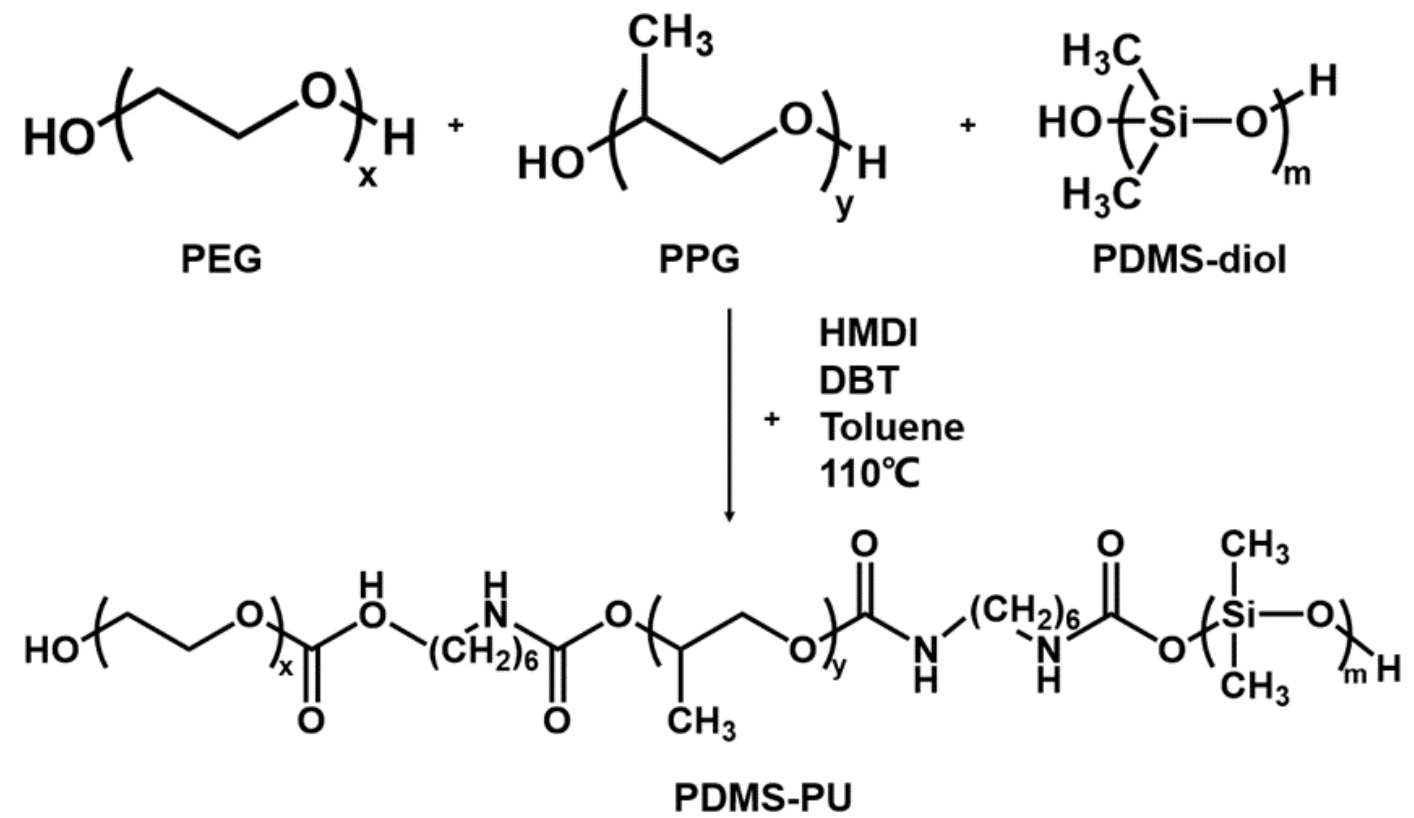

Figure S1. Synthetic scheme of PDMS-PU.

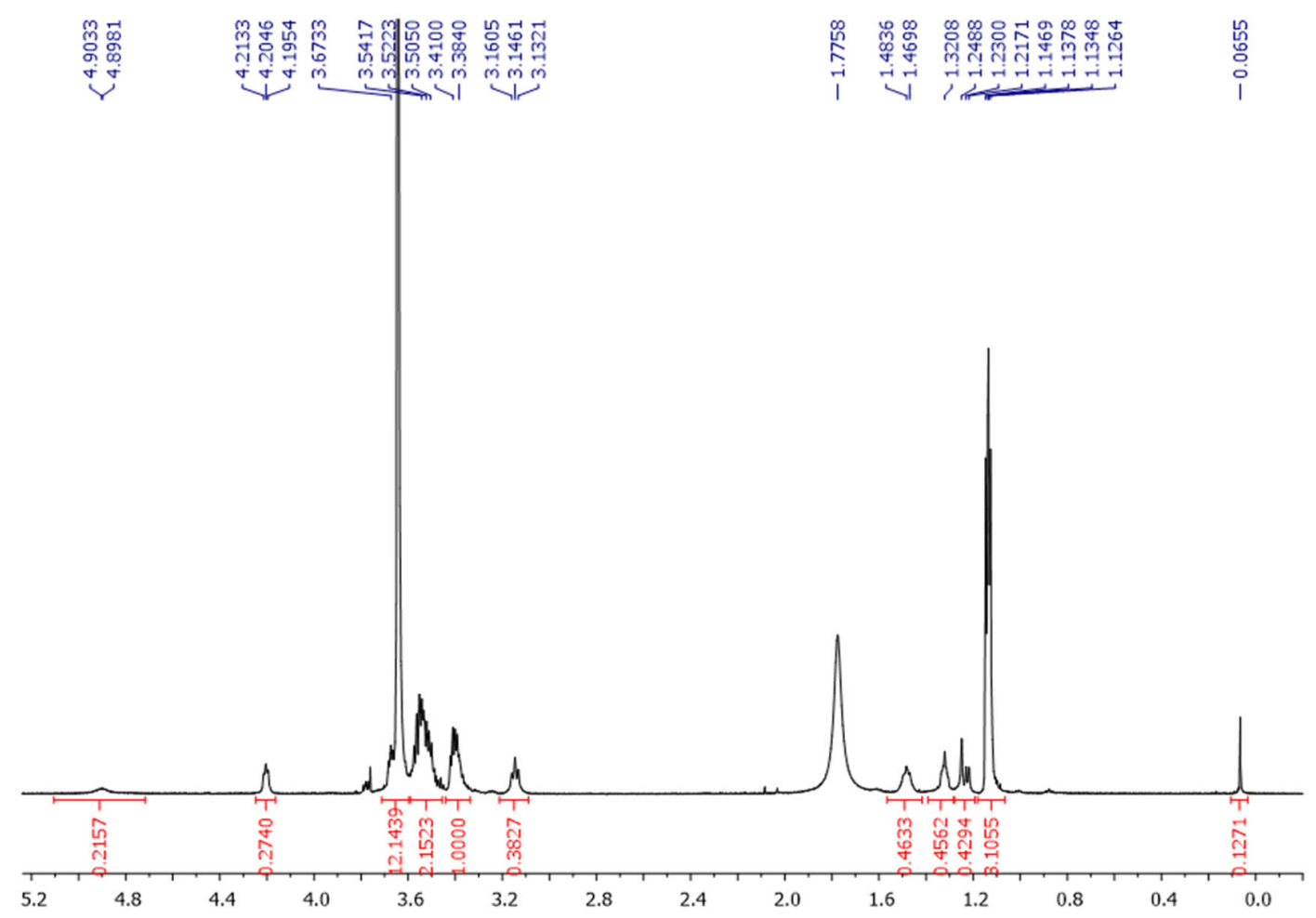

Figure S2. ${ }^{1} \mathrm{H}$ NMR spectrum of PDMS-PU. 


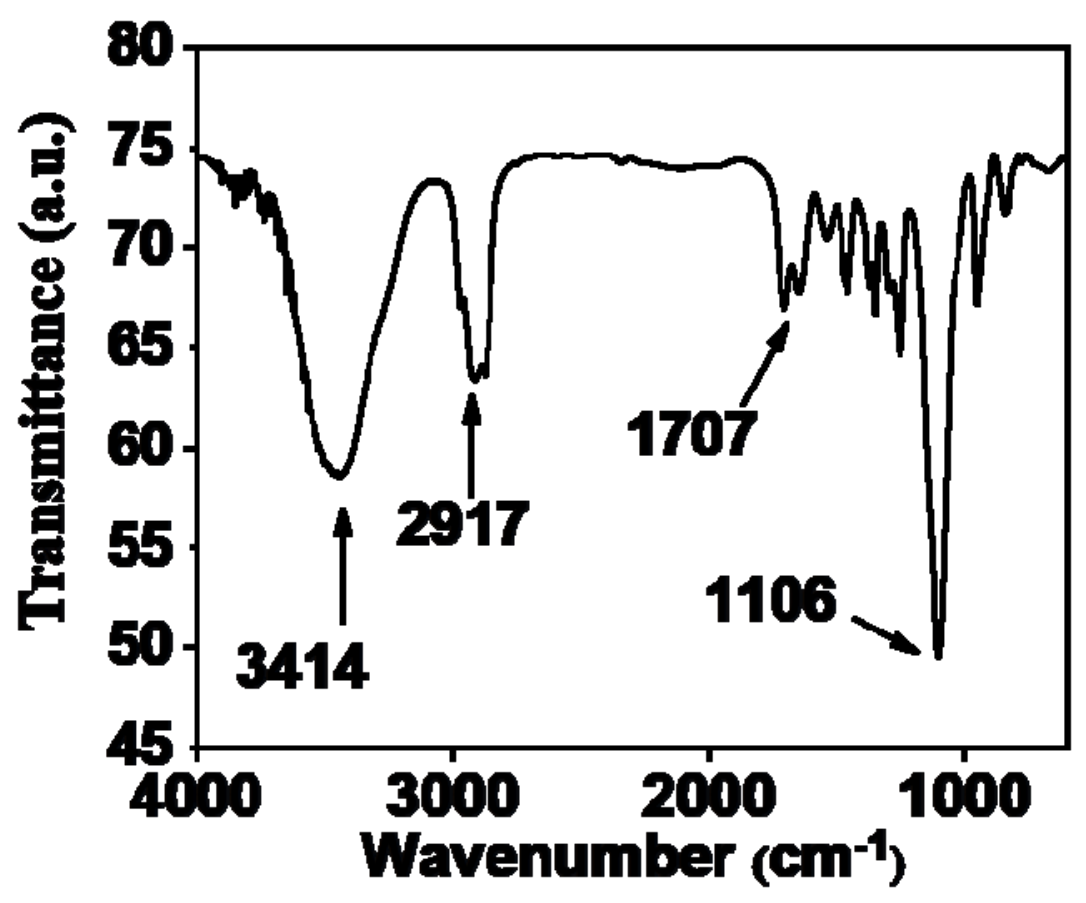

Figure S3. The FT-IR spectrum of PDMS-PU.

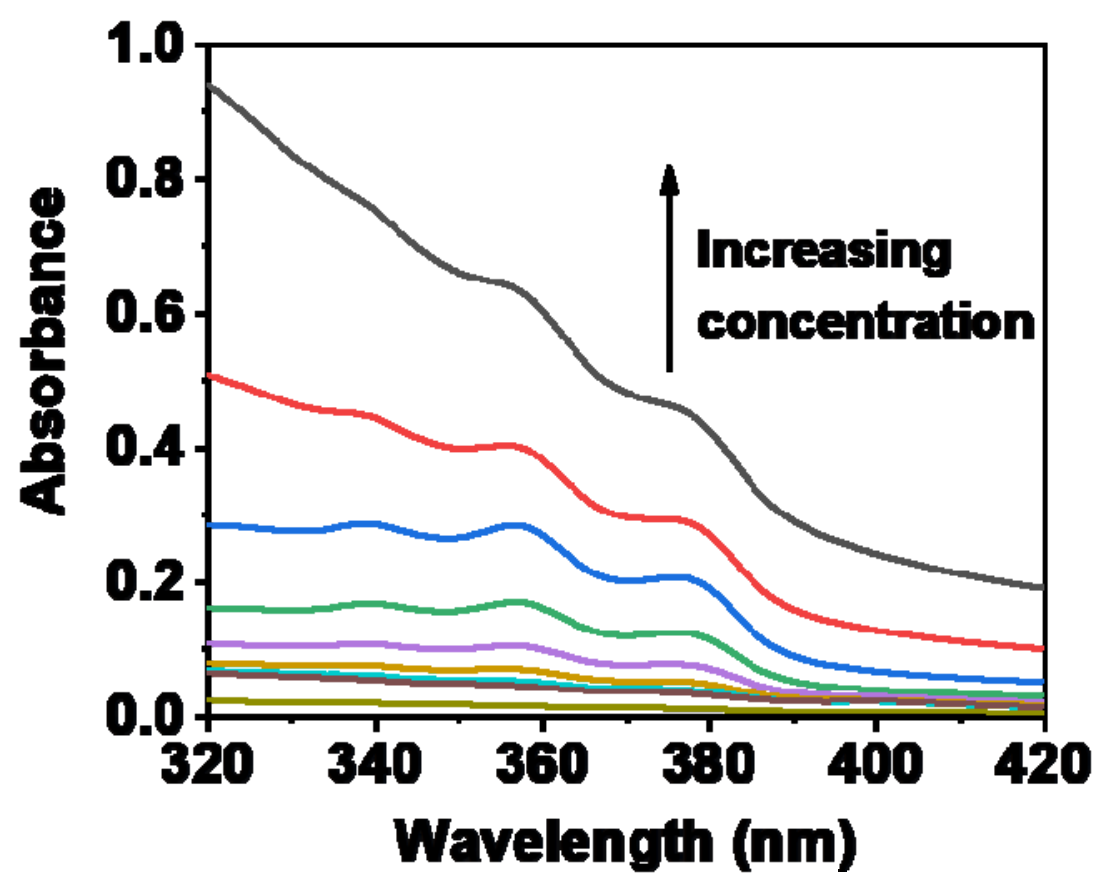

Figure S4. UV-Vis spectra of DPH with increasing concentration of lignin-incorporated thermogel in water at $25^{\circ} \mathrm{C}$. 


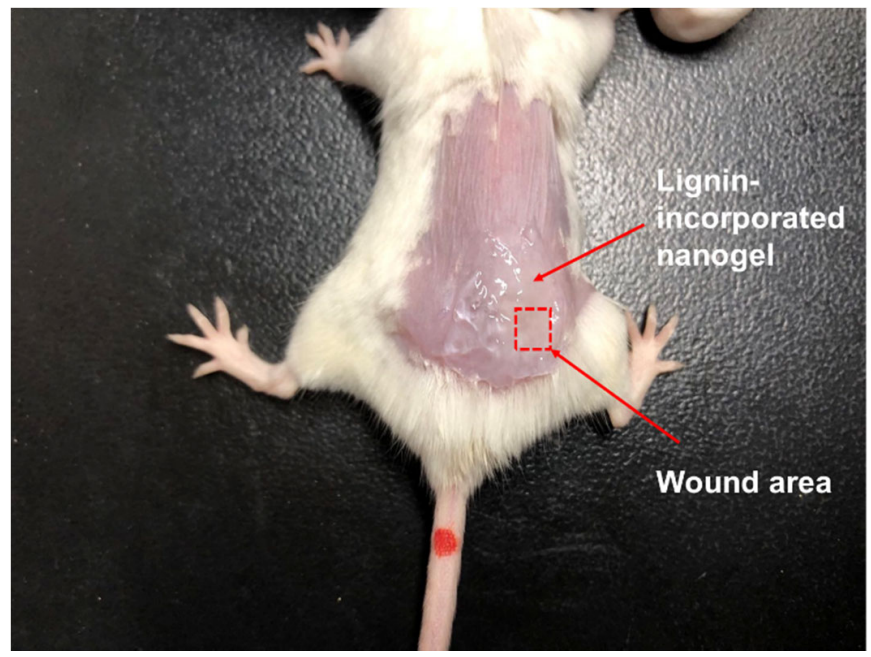

Figure S5. A photo showing how the nanogel was initially applied as a wound dressing.

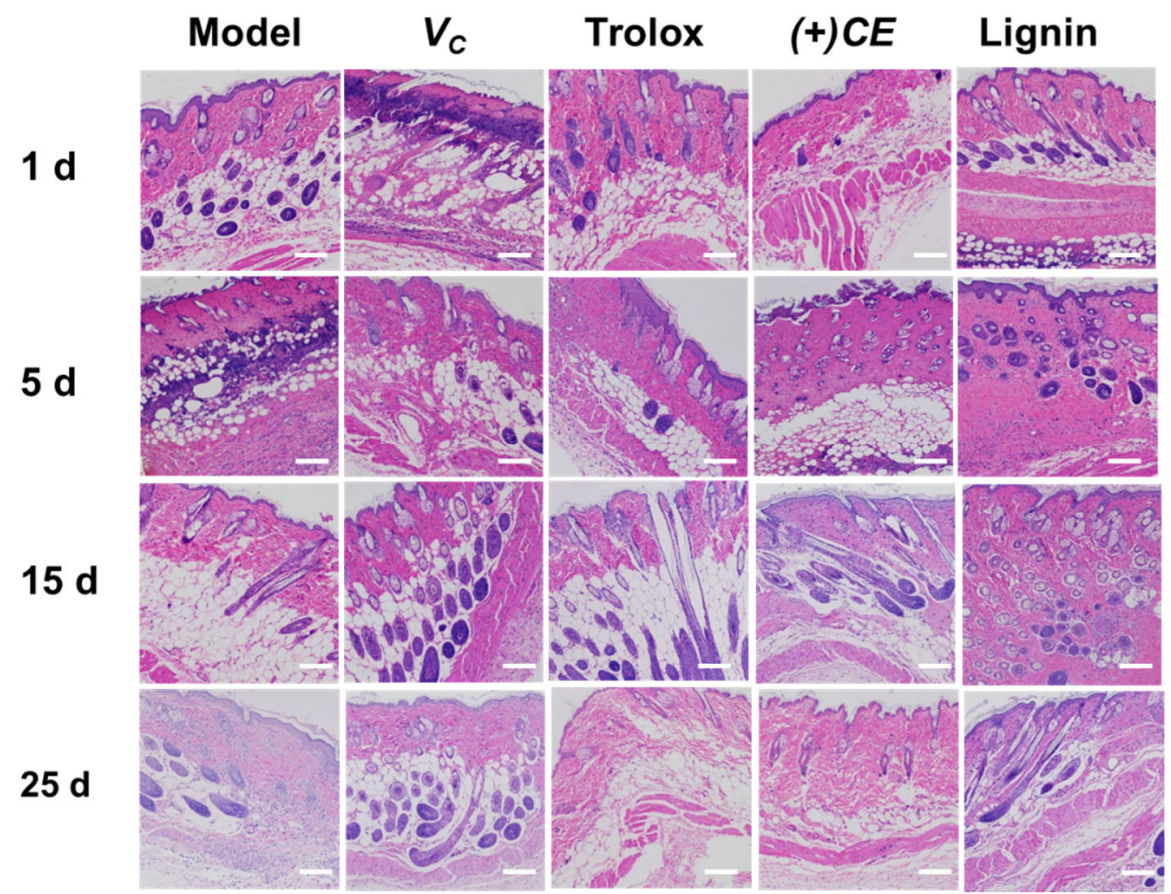

Figure S6. H\&E stained images of the mice granulation tissue treated with $\mathrm{V}_{\mathrm{C}}$, Trolox, $(+) \mathrm{CE}$, and lignin group on day 1, 5, 15, and 25, respectively. Scale bar: $200 \mu \mathrm{m}$. 